\title{
Guidance and leakage properties of chiral optical fibers
}

\author{
Fernando M. Janeiro, Carlos R. Paiva, and António L. Topa \\ Instituto de Telecomunicações, Instituto Superior Técnico, Technical University of Lisbon, Av. Rovisco Pais, 1 , \\ 1049-001 Lisboa, Portugal
}

Received November 12, 2001; revised manuscript received April 30, 2002

\begin{abstract}
The field theory of guided waves in optical fibers with step-index profiles and in which both core and cladding are chiral isotropic media is developed. We show that both surface and semileaky modes can propagate in optically active fibers. To shed light on the guidance and leakage properties of chiral isotropic fibers we present a physical interpretation and several numerical results. The new leakage effect associated with semileaky modes is an important property that cannot be neglected in the analysis of chiral optical fibers but that, nevertheless, has been systematically disregarded in the literature. (c) 2002 Optical Society of America

OCIS codes: 260.2110, 060.2400, 350.5500, 060.2290.
\end{abstract}

\section{INTRODUCTION}

Chiral or optically active media were discovered at the beginning of the 19 th $^{\text {century. }}{ }^{1}$ Although natural chiral materials are available at optical frequencies, it was shown at the beginning of the 20th century that chirality can be experimentally observed at microwave frequencies. $^{2}$ More-recent studies of the electromagnetic properties of chiral media ${ }^{3}$ have prompted a renewed interest in this subject by the scientific community. One can achieve an artificial chiral isotropic material by randomly inserting a small number of chiral objects (such as helices) of the same handedness into the host isotropic medium. ${ }^{3}$ Artificial chiral materials are already available for suboptical frequencies. ${ }^{4}$ Recent advances in material science, namely, in polymers and composite materials, have provided good reasons to believe that artificially produced chiral media will soon be available for optical frequencies.

A good account of the boom in research in electromagnetic theory and applications of chiral media until 1994 was provided by the overview in Ref. 5. An extensive discussion of wave propagation in waveguides filled with chiral media, the so-called chirowaveguides, can be found in the literature (see, e.g., Ref. 6 and references therein). Open dielectric chirowaveguides are well suited for applications at optical and suboptical frequencies. ${ }^{7-12}$ Circular open chirowaveguides, in particular, have been also analyzed. ${ }^{13-18}$ One would say, therefore, that the field theory of guided waves in chiral optical fibers, including the general case in which both core and cladding are made from chiral materials, ${ }^{16}$ appears already to have been covered in the literature. We show in this paper that such is not the case: There has been no study of the leakage effect associated with semileaky modes in chiral optical fibers, as far as the authors are aware.

It was already shown in Ref. 11 that, in planar dielectric chirowaveguides, semileaky modes can propagate. We show in this paper that semileaky modes can also propagate in chiral optical fibers with step-index profiles.
This new leakage effect is an essential feature for the correct and complete characterization of the guidance properties of chiral optical fibers. In a previous communication by the present authors ${ }^{19}$ it was shown that, in a chiral optical fiber in which both core and cladding are chiral, semileaky modes can propagate. However, we believe that this is the first full-length paper in an archival journal in which the analytical theory along with a detailed physical interpretation of the guidance and leakage properties of chiral optical fibers, including several numerical results for surface and semileaky modes, is presented. We should stress that leakage is an important issue that cannot be neglected when one is analyzing hybrid modes in chiral optical fibers but that nevertheless has been systematically disregarded in the literature so far.

The leakage properties of a class of open dielectric waveguides, with potential interest for integrated optics and microwave integrated circuits, were studied by Peng and Oliner. ${ }^{20}$ The leakage effect was due to geometry, namely, to the TE-TM coupling that occurs at the step discontinuities on the lateral walls of the guide's central dielectric strip. ${ }^{21}$ Another kind of leakage was extensively analyzed, both theoretically and experimentally, in anisotropic metal-diffused optical waveguides. ${ }^{22-34}$ In such anisotropic waveguides, one of the two characteristic waves in the substrate ceases to be totally internally reflected at the film-substrate interface, thus leading to energy radiation into the substrate region. A similar effect can be found in asymmetric chiroslab guides in which both film and substrate are made from chiral materials. ${ }^{11}$ All these leakage effects-which are due to geometry, anisotropy, or chirality-always occur in guided (or, more rigorously, semiguided) modes; i.e., these are not completely unguided leaky waves that can be included in the electromagnetic field only in special representations, as in the steepest-descent representation, namely, in excitation problems. ${ }^{35}$

The theory of electromagnetic wave propagation in isotropic chiral media differs from the most common aspects 
of isotropic achiral media. In fact, for optically active media, one should introduce-according to Condon ${ }^{36}$ - the gyrotropic parameter $g$ in the constitutive relations written in the time domain, as follows:

$$
\begin{aligned}
& \mathcal{D}=\epsilon_{0} \epsilon \mathcal{E}-g \frac{\partial \mathcal{H}}{\partial t}, \\
& \mathcal{B}=\mu_{0} \mu \mathcal{H}+g \frac{\partial \mathcal{E}}{\partial t} .
\end{aligned}
$$

The gyrotropic parameter is responsible for optical activity. When we write these equations in the frequency domain, by using the Fourier transform $\mathbf{A}(\mathbf{r}, \omega)$ of $\mathcal{A}(\mathbf{r}, t)$ such that

$$
\begin{aligned}
& \mathbf{A}(\mathbf{r}, \omega)=\int_{-\infty}^{\infty} \mathcal{A}(\mathbf{r}, t) \exp (i \omega t) \mathrm{d} t \\
& \mathcal{A}(\mathbf{r}, t)=\frac{1}{2 \pi} \int_{-\infty}^{\infty} \mathbf{A}(\mathbf{r}, \omega) \exp (-i \omega t) \mathrm{d} \omega
\end{aligned}
$$

we obtain from Eqs. (1)

$$
\left[\begin{array}{l}
\mathbf{D} \\
\mathbf{B}
\end{array}\right]=\left[\begin{array}{cc}
\epsilon_{0} \boldsymbol{\epsilon} & i \sqrt{\epsilon_{0} \mu_{0} \chi} \\
-i \sqrt{\epsilon_{0} \mu_{0} \chi} & \mu_{0} \mu
\end{array}\right]\left[\begin{array}{l}
\mathbf{E} \\
\mathbf{H}
\end{array}\right],
$$

with

$$
\epsilon_{0} Z_{0}=\mu_{0} Y_{0}=\sqrt{\epsilon_{0} \mu_{0}}=1 / c,
$$

where $c$ is the velocity of light in vacuum and where the normalized chirality parameter $\chi=\chi(\omega)$, such that

$$
\chi(\omega)=\frac{\omega g}{\sqrt{\epsilon_{0} \mu_{0}}}=k_{0} c^{2} g=\frac{2 \pi c^{2}}{\lambda} g,
$$

has been introduced. The chirality parameter, which is responsible for optical activity and spatial dispersion, is, in fact, an odd function of frequency: According to Eqs. (1), optical activity vanishes in the steady-state regime. Although several models for the constitutive relations have been used in the literature, we use Eq. (3) henceforth as our model for chiral media. The existence of such model diversity ${ }^{37}$ makes it necessary to begin any study of chiral electromagnetics by clearly stating what model has been adopted by the authors.

We should note, however, that Eq. (3) is a reciprocal model for bi-isotropic media that is consistent with the socalled Drude-Born-Fedorov model ${ }^{38}$ : As Lakhtakia and Weiglhofer ${ }^{39-42}$ have pointed out, if one uses the Post constraint, ${ }^{43}$ then nonreciprocal bi-isotropic media (particularly the so-called Tellegen medium) do not exist-a controversial statement that can be contested. ${ }^{44-46}$ According to the Post constraint, in Minkowski's representation of electromagnetics the alternation (a determinantlike operation) of the four-dimensional constitutive tensor should vanish. ${ }^{43}$ This constraint eliminates a redundancy that emerges when the linear constitutive relations are substituted into Maxwell's equations. ${ }^{42}$ We should stress, however, that this redundancy is intimately related to the fact that $\mathbf{E}$ and $\mathbf{B}$ should be considered primitive fields. We adopted in Eq. (3) the commonly used convention of expressing $\mathbf{D}$ and $\mathbf{B}$ in terms of $\mathbf{E}$ and $\mathbf{H}$, albeit the correct physical picture should express induction fields $\mathbf{D}$ and $\mathbf{H}$ as functions of actual electric and magnetic fields $\mathbf{E}$ and B. Although we adhere to the Post constraint, as it has never been known to be violated by a physical medium, we cannot agree with the rhetorical argument that a nonreciprocal bi-isotropic medium is just an oxymoron; see, e.g., Eq. (2.153) of Ref. 47. Of course the ultimate scientific criterion is experimental evidence. Therefore we are willing to review our opinion if a recognizable nonreciprocal bi-isotropic medium can be presented.

A chiral isotropic medium is then characterized by three parameters $(\epsilon, \mu, \chi)$, in counterpoint to achiral isotropic media, which are characterized by two parameters $(\epsilon, \mu)$ only. From the designer's point of view the most important property of chiral devices is chirality parameter $\chi$, which provides an extra degree of freedom, thus leading to a more-flexible optimization procedure.

According to the standard terminology of modern electromagnetics, Maxwell's equations should be written exclusively in terms of primitive fields $\mathbf{E}$ and $\mathbf{B}$. However, in this paper we adopt the commonly used convention of writing Maxwell's equations in accordance with Eq. (3). Hence, in the frequency domain and for source-free regions (where $\overline{\mathbf{I}}$ is the unit dyadic),

$$
\left[\begin{array}{cc}
\nabla \times \overline{\mathbf{I}} & \overline{\mathbf{0}} \\
\overline{\mathbf{0}} & -\nabla \times \overline{\mathbf{I}}
\end{array}\right]\left[\begin{array}{l}
\mathbf{E} \\
\mathbf{H}
\end{array}\right]=i \omega\left[\begin{array}{l}
\mathbf{B} \\
\mathbf{D}
\end{array}\right]
$$

It can easily be shown that, in unbounded chiral media, there are two TEM characteristic waves: a right-hand circularly polarized (RCP) wave and a left-hand circularly polarized (LCP) wave, and hence in optically active media the polarization plane of linearly polarized light rotates with propagation. ${ }^{47}$ In an optical fiber in which both core and cladding are chiral, a surface wave can propagate as long as the core's two characteristic waves undergo total internal reflection at the core-cladding interface. However, if one of the core's two characteristic waves ceases to be totally internally reflected at the core-cladding interface while the other characteristic wave still undergoes total internal reflection, a semileaky wave will result. Hence, in semileaky waves, leakage is due to energy that is continuously radiated outward by the core's characteristic wave that is transmitted. When both guidance and leakage are present, there is always a core's characteristic wave that is not transmitted; that is why the resultant hybrid mode is designated a semileaky wave. Therefore, for a given hybrid mode, the longitudinal wave number will be a real number for surface waves and a complex number for semileaky waves. In fact, for semileaky waves, the real part of the longitudinal wave number has to account for guidance, whereas the imaginary part has to account for the energy radiated through leakage.

This paper is organized as follows: In Section 2 the analytical framework is derived. Next, a physical interpretation for both surface and semileaky modes is developed, (Section 3). Then in Section 4 the numerical results for both surface and semileaky modes in chiral optical fibers are presented. Finally, in Section 5, our conclusions are briefly outlined. 


\section{ANALYTICAL FORMULATION}

In this section we present our analytical approach to the field theory of guided waves in chiral optical fibers. The modal equation for surface and semileaky waves in chiral optical fibers is derived. The general case, depicted in Fig. 1, for which both core and cladding are chiral media-characterized, respectively, by $\left(\epsilon_{1}, \mu_{1}, \chi_{1}\right)$ and $\left(\epsilon_{2}, \mu_{2}, \chi_{2}\right)$-is considered. Let us adopt cylindrical coordinates $(r, \phi, z)$ and, for the guided modes in the chiral fiber, a field variation $\exp [i(m \phi+\beta z-\omega t)]$, where $m$ and $\beta$ are the azimuthal and the longitudinal wave numbers, respectively. We then obtain the modal equation that provides the relation between frequency $\omega$ and longitudinal wave number $\beta$ by enforcing the boundary conditions (i.e., the continuity of the tangential components of $\mathbf{E}$ and $\mathbf{H}$ across the core-cladding interface).

If we consider a general chiral region characterized by $(\epsilon, \mu, \chi)$, then from Eqs. (3) and (6) we get

$$
\begin{aligned}
E_{r}= & -\frac{i}{\beta} \frac{\partial E_{z}}{\partial r}+\frac{k_{0}}{\beta D}\left[Z_{0} \mu\left(k_{+} k_{-}-\beta^{2}\right) g\right. \\
& \left.+i \chi\left(k_{+} k_{-}+\beta^{2}\right) f\right], \\
H_{r}= & -\frac{i}{\beta} \frac{\partial H_{z}}{\partial r}-\frac{k_{0}}{\beta D}\left[Y_{0} \epsilon\left(k_{+} k_{-}-\beta^{2}\right) f\right. \\
& \left.-i \chi\left(k_{+} k_{-}+\beta^{2}\right) g\right],
\end{aligned}
$$

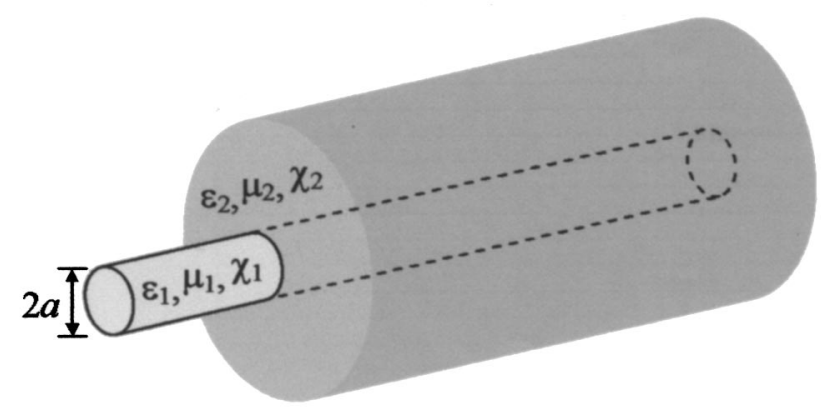

(a)

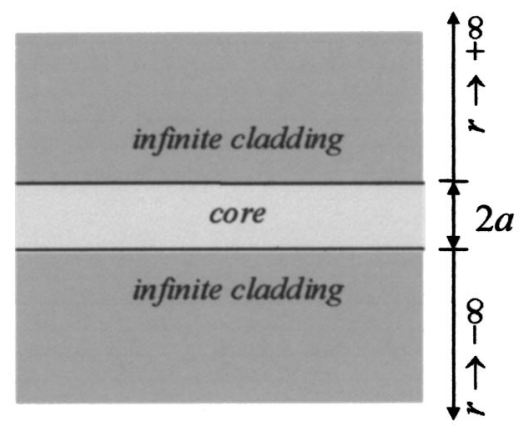

(b)

Fig. 1. (a) Chiral optical fiber. The cladding has an infinite radius. (b) Longitudinal cross section.

$$
\begin{aligned}
& E_{\phi}=\frac{1}{D}\left(S f-2 i k_{0}{ }^{2} Z_{0} \mu \chi g\right), \\
& H_{\phi}=\frac{1}{D}\left(S g+2 i k_{0}{ }^{2} Y_{0} \epsilon \chi f\right),
\end{aligned}
$$

where

$$
\begin{aligned}
k_{ \pm} & =(\sqrt{\epsilon \mu} \pm \chi) k_{0} \\
S & =\left(\epsilon \mu+\chi^{2}\right) k_{0}{ }^{2}-\beta^{2} \\
D & =\left(k_{+}{ }^{2}-\beta^{2}\right)\left(k_{-}^{2}-\beta^{2}\right) \\
f & =-m \frac{\beta}{r} E_{z}-i k_{0} Z_{0} \mu \frac{\partial H_{z}}{\partial r}-k_{0} \chi \frac{\partial E_{z}}{\partial r} \\
g & =-m \frac{\beta}{r} H_{z}+i k_{0} Y_{0} \epsilon \frac{\partial E_{z}}{\partial r}-k_{0} \chi \frac{\partial H_{z}}{\partial r} .
\end{aligned}
$$

One should note that $k_{ \pm}$in Eq. (8a) are the wave numbers for the RCP and the LCP characteristic waves in unbounded chiral media. When we define

$$
\kappa_{ \pm}^{2}=k_{ \pm}^{2}-\beta^{2}
$$

then $S=\left(\kappa_{+}{ }^{2}+\kappa_{-}{ }^{2}\right) / 2$ and $D=\kappa_{+}{ }^{2} \kappa_{-}{ }^{2}$. In Eq. (9) $\kappa_{+}$ represent the radial wave numbers associated with each characteristic wave. By using the so-called Bohren decomposition $^{48}$ we may write

$$
\left[\begin{array}{l}
E_{z} \\
H_{z}
\end{array}\right]=\overline{\mathbf{M}}\left[\begin{array}{l}
E_{z}{ }^{(+)} \\
E_{z}{ }^{(-)}
\end{array}\right], \quad \overline{\mathbf{M}}=\left[\begin{array}{cc}
1 & 1 \\
-i Y_{c} & i Y_{c}
\end{array}\right],
$$

with $Y_{c}=y_{c} Y_{0}=\sqrt{\epsilon / \mu} Y_{0}$. Functions $E_{z}^{( \pm)}(r, \phi, z, t)$ are such that $\nabla^{2} E_{z}{ }^{( \pm)}+\kappa_{ \pm}{ }^{2} E_{z}{ }^{( \pm)}=0$ or, if one takes $E_{z}^{( \pm)}(r, \phi, z, t)=\psi_{ \pm}(r) \exp (i m \phi) \exp [i(\beta z-\omega t)]$, then

$$
\frac{\partial^{2} \psi_{ \pm}}{\partial r^{2}}+\frac{1}{r} \frac{\partial \psi_{ \pm}}{\partial r}+\left({\kappa_{ \pm}}^{2}-\frac{m^{2}}{r^{2}}\right) \psi_{ \pm}=0 .
$$

If $a$ is the core radius and

$$
\begin{aligned}
& h_{ \pm}{ }^{2}={\beta_{ \pm}}^{2}-\beta^{2}, \quad \alpha_{ \pm}{ }^{2}=\beta^{2}-{\gamma_{ \pm}}^{2}, \\
& \beta_{ \pm}=p_{ \pm} k_{0}, \quad p_{ \pm}=n_{1} \pm \chi_{1}, \\
& \gamma_{ \pm}=q_{ \pm} k_{0}, \quad q_{ \pm}=n_{2} \pm \chi_{2}, \\
& n_{1}=\sqrt{\epsilon_{1} \mu_{1}}, \quad n_{2}=\sqrt{\epsilon_{2} \mu_{2}},
\end{aligned}
$$

then, for $r \leqslant a$,

$$
\frac{\partial^{2} \psi_{ \pm}}{\partial r^{2}}+\frac{1}{r} \frac{\partial \psi_{ \pm}}{\partial r}+\left(h_{ \pm}{ }^{2}-\frac{m^{2}}{r^{2}}\right) \psi_{ \pm}=0,
$$

which are Bessel differential equations, whereas, for $r>a$,

$$
\frac{\partial^{2} \psi_{ \pm}}{\partial r^{2}}+\frac{1}{r} \frac{\partial \psi_{ \pm}}{\partial r}-\left({\alpha_{ \pm}}^{2}+\frac{m^{2}}{r^{2}}\right) \psi_{ \pm}=0,
$$

which are modified Bessel differential equations. Furthermore, if we choose

$$
\left[\begin{array}{l}
E_{z}(r, \phi, z, t) \\
H_{z}(r, \phi, z, t)
\end{array}\right]=\left[\begin{array}{l}
F(r) \\
G(r)
\end{array}\right] \exp (i m \phi) \exp [i(\beta z-\omega t)]
$$

then 


$$
\left[\begin{array}{l}
F(r) \\
G(r)
\end{array}\right]=\overline{\mathbf{M}}\left[\begin{array}{l}
\psi_{+}(r) \\
\psi_{-}(r)
\end{array}\right], \quad \psi_{ \pm}(r)=\left\{\begin{array}{ll}
A_{ \pm} J_{m}\left(h_{ \pm} r\right) & r \leqslant a \\
B_{ \pm} K_{m}\left(\alpha_{ \pm} r\right) & r>a
\end{array} .\right.
$$

Because the electromagnetic field must be finite for $r$ $=0$, Bessel functions of the second kind $Y_{m}\left(h_{ \pm} r\right)$ were disregarded as solutions of Eqs. (13). Likewise, modified Bessel functions of the first kind $I_{m}\left(\alpha_{+} r\right)$ were also disregarded as solutions of Eqs. (14) because, for surface waves, the electromagnetic field should vanish when $r \rightarrow \infty$. Hence, in the chiral core $(r \leqslant a)$,

$$
\left[\begin{array}{l}
F(r) \\
G(r)
\end{array}\right]=\overline{\mathbf{M}}\left[\begin{array}{l}
A_{+} J_{m}\left(h_{+} r\right) \\
A_{-} J_{m}\left(h \_r\right)
\end{array}\right]
$$

where $Y_{c}=Y_{1}=n_{1} Y_{0} / \mu_{1}$. Similarly, in the chiral cladding $(r>a)$,

$$
\left[\begin{array}{l}
F(r) \\
G(r)
\end{array}\right]=\overline{\mathbf{M}}\left[\begin{array}{l}
B_{+} K_{m}\left(\alpha_{+} r\right) \\
B \_K_{m}\left(\alpha_{-} r\right)
\end{array}\right]
$$

where $Y_{c}=Y_{2}=n_{2} Y_{0} / \mu_{2}$. Let us define the following normalized variables:

$$
u_{ \pm}=h_{ \pm} a, \quad w_{ \pm}=\alpha_{ \pm} a .
$$

Accordingly, we have $u_{ \pm}^{2}+w_{ \pm}^{2}=v_{ \pm}^{2}$, with $v_{ \pm}^{2}$ $=\left(\beta_{ \pm} a\right)^{2}-\left(\gamma_{ \pm} a\right)^{2}$. Then, if

$$
\eta=\frac{Y_{2}}{Y_{1}}=\frac{y_{2}}{y_{1}}=\frac{\mu_{1} n_{2}}{\mu_{2} n_{1}},
$$

we get, after imposing the first two boundary conditions (the continuity of $E_{z}$ and $H_{z}$ at $r=a$ ):

$$
\begin{aligned}
{\left[\begin{array}{rr}
(1+\eta) K_{m}\left(w_{+}\right) & (1-\eta) K_{m}\left(w_{-}\right) \\
(1-\eta) K_{m}\left(w_{+}\right) & (1+\eta) K_{m}\left(w_{-}\right)
\end{array}\right]\left[\begin{array}{l}
B_{+} \\
B_{-}
\end{array}\right] } \\
=\left[\begin{array}{l}
2 A_{+} J_{m}\left(u_{+}\right) \\
2 A_{-} J_{m}\left(u_{-}\right)
\end{array}\right],
\end{aligned}
$$

from which we further obtain

$$
\begin{array}{ll}
B_{+}=Q_{+} A_{+}+Q_{-} A_{-}, & B_{-}=R_{+} A_{+}+R A_{-}, \\
Q_{ \pm}=\frac{(\eta \pm 1) J_{m}\left(u_{ \pm}\right)}{2 \eta K_{m}\left(w_{+}\right)}, & R_{ \pm}=\frac{(\eta \mp 1) J_{m}\left(u_{ \pm}\right)}{2 \eta K_{m}\left(w_{-}\right)} .
\end{array}
$$

Therefore, after imposing the other two boundary conditions (the continuity of $E_{\phi}$ and $H_{\phi}$ at $r=a$ ), we get

$$
\left[\begin{array}{ll}
\Lambda_{11} & \Lambda_{12} \\
\Lambda_{21} & \Lambda_{22}
\end{array}\right]\left[\begin{array}{l}
A_{+} \\
A_{-}
\end{array}\right]=\left[\begin{array}{l}
0 \\
0
\end{array}\right]
$$

with

$$
\begin{array}{ll}
\Lambda_{11}=\Gamma_{+}-\Delta_{+}, & \Lambda_{12}=\Gamma_{-}-\Delta_{-}, \\
\Lambda_{21}=i \Gamma_{+}-\Omega_{+}, & \Lambda_{22}=-i \Gamma_{-}-\Omega_{-} .
\end{array}
$$

We have introduced

$$
\begin{aligned}
& \Gamma_{ \pm}=2\left(a_{1} \mp i y_{1} a_{2}\right) \rho_{ \pm}+2\left(a_{3} \mp i y_{1} a_{4}\right) \sigma_{ \pm} \\
& \Delta_{ \pm}=2\left(b_{1} \mp i y_{1} b_{2}\right) \rho_{ \pm}-\left(b_{3} \zeta_{ \pm}-i y_{2} b_{4} \xi_{ \pm}\right) \\
& \Omega_{ \pm}=2\left(\eta y_{2} b_{2} \pm i b_{1}\right) \rho_{ \pm}-\eta\left(y_{2} b_{4} \zeta_{ \pm}+i b_{3} \xi_{ \pm}\right)
\end{aligned}
$$

as well as the auxiliary coefficients

$$
\begin{aligned}
& \rho_{ \pm}=J_{m}\left(u_{ \pm}\right), \quad \sigma_{ \pm}=u_{ \pm} J_{m}^{\prime}\left(u_{ \pm}\right), \\
& \tau_{ \pm}=w_{ \pm} \frac{K_{m}^{\prime}\left(w_{ \pm}\right)}{K_{m}\left(w_{ \pm}\right)}, \\
& \zeta_{ \pm}=-\left[\left(\tau_{+}+\tau_{-}\right) \pm \frac{1}{\eta}\left(\tau_{+}-\tau_{-}\right)\right] \rho_{ \pm}, \\
& \xi_{ \pm}=-\left[\left(\tau_{+}-\tau_{-}\right) \pm \frac{1}{\eta}\left(\tau_{+}+\tau_{-}\right)\right] \rho_{ \pm} .
\end{aligned}
$$

Moreover, coefficients $a_{i}$ and $b_{i}$ (with $i=1,2,3,4$ ) in Eqs. (25) are given by

$$
\begin{aligned}
& a_{1}=-\frac{m(\beta a)}{2}\left(\frac{1}{u_{+}{ }^{2}}+\frac{1}{u_{-}{ }^{2}}\right), \\
& b_{1}=\frac{m(\beta a)}{2}\left(\frac{1}{w_{+}{ }^{2}}+\frac{1}{w_{-}{ }^{2}}\right), \\
& a_{2}=2 i m \mu_{1} \chi_{1} \frac{(\beta a)\left(k_{0} a\right)^{2}}{u_{+}{ }^{2} u_{-}{ }^{2}}, \\
& b_{2}=2 i m \mu_{2} \chi_{2} \frac{(\beta a)\left(k_{0} a\right)^{2}}{w_{+}{ }^{2} w_{-}{ }^{2}}, \\
& a_{3}=\chi_{1}\left(k_{0} a\right) \frac{\left(\beta_{+} \beta_{-}+\beta^{2}\right) a^{2}}{u_{+}{ }^{2} u_{-}{ }^{2}}, \\
& b_{3}=\chi_{2}\left(k_{0} a\right) \frac{\left(\gamma_{+} \gamma_{-}+\beta^{2}\right) a^{2}}{w_{+}{ }^{2} w_{-}{ }^{2}}, \\
& a_{4}=-i \mu_{1}\left(k_{0} a\right) \frac{\left(\beta_{+} \beta_{-}{ }^{2} \beta^{2}\right) a^{2}}{u_{+}{ }^{2} u_{-}{ }^{2}}, \\
& b_{4}=-i \mu_{2}\left(k_{0} a\right) \frac{\left(\gamma_{+} \gamma_{-}-\beta^{2}\right) a^{2}}{w_{+}{ }^{2} w_{-}{ }^{2}} .
\end{aligned}
$$

Finally, for nontrivial solutions, we obtain from Eq. (23)

$$
\Lambda_{11} \Lambda_{22}-\Lambda_{12} \Lambda_{21}=0 .
$$

This is the modal equation for an optical fiber for which both core and cladding are chiral media. Because of the magnetoelectric nature of chiral media, only hybrid modes will propagate: There are no $\mathrm{TE}_{0 n}$ or $\mathrm{TM}_{0 n}$ modes in chiral optical fibers as in common isotropic fibers. ${ }^{49,50}$ One should note that, for surface modes, $\alpha_{ \pm}>0$ and hence $w_{ \pm}>0$. For semileaky modes, however, $\beta$ is complex; hence $h_{ \pm}, u_{ \pm}, \alpha_{ \pm}$, and $w_{ \pm}$are complex as well. For a common isotropic (or achiral) fiber, one has $\chi_{1}=\chi_{2}=0$. One can easily show that, for this special case, Eq. (28) reduces to Eq. (7.55) of Ref. 49.

\section{PHYSICAL DISCUSSION}

In this section we present a physical discussion of surface and semileaky modes in chiral optical fibers. For simplicity we consider the core-cladding interface to be planar. Moreover, in everything that follows we always assume that core and cladding have the same chirality, i.e., that $\chi_{1}=\chi_{2}=\chi$, with $\chi>0$. Also, we consider that the 
core is denser than the cladding, so $p_{+}=n_{1}+\chi>q_{+}$ $=n_{2}+\chi$; hence we should always have

$$
n_{1}=\sqrt{\epsilon_{1} \mu_{1}}>n_{2}=\sqrt{\epsilon_{2} \mu_{2}} .
$$

Owing to the magnetoelectric nature of a chiral medium, there are two characteristic waves in the chiral cladding. Therefore, according to our simplifying hypothesis that the core-cladding interface is planar, we have to analyze the reflection problem at a planar interface between two chiral half-spaces. Accordingly, the RCP and LCP characteristic waves are uncoupled. One should stress, however, that, in the real situation, these two characteristic waves are coupled, as they actually form the hybrid mode altogether. When both of the core's characteristic waves undergo total internal reflection at the core-cladding interface, a surface mode is propagating; otherwise, at least one of the core's two characteristic waves is transmitted into the cladding and hence leakage will occur. However, there are two different situations: When only one of the core's two characteristic waves is transmitted, a semileaky mode is still propagating along the fiber; when both of the core's characteristic waves are transmitted, then we no longer have a propagating mode. A semileaky mode is a guided (or, more rigorously, a semiguided) wave that, simultaneously, is leaking energy. This can be true only if longitudinal wave number $\beta$ is a complex number, such that

$$
\beta=n_{\mathrm{eff}} k_{0}+i(\alpha / 2),
$$

where $n_{\text {eff }}$ is the effective refractive index that arises from guidance and $\alpha$ is the power loss coefficient that arises from leakage. A surface mode is a particular case of Eq. (30), with $\alpha=0$ and $\beta=n_{\text {eff }} k_{0}$.

For the planar interface two different cases have to be considered: when the incident wave is the core's RCP wave and when it is the core's LCP wave. In any case, the continuity of the tangential field components at the interface leads to Snell's law. Hence, when the incident wave is the core's RCP characteristic wave, one should have

$$
\begin{aligned}
\beta=\beta_{+} \sin \theta_{1}^{(+)} & =\beta_{-} \sin \theta_{1}^{(+,-)} \\
& =\gamma_{+} \sin \theta_{2}^{(+,+)}=\gamma_{-} \sin \theta_{2}{ }^{(+,-)},
\end{aligned}
$$

as depicted in Fig. 2(a) and where $\theta_{1}{ }^{(+)}=\theta_{1}{ }^{\left(+,{ }^{+}\right)}$(the first sign in the superscript refers to the incident characteristic wave and the second sign refers to the characteristic wave under consideration). As we are considering oblique incidence, a reflected wave with polarization orthogonal to the polarization of the incident wave (i.e., a LCP wave) will also appear in the core. Accordingly, we will have two radial wave numbers in the core: $h_{+}$ $=\beta_{+} \cos \theta_{1}^{(+,+)}$for the core's RCP (incident and reflected) wave and $h_{-}=\beta_{-} \cos \theta_{1}^{(+,-)}$for the core's LCP (reflected) wave. In the cladding, however, the two radial wave numbers will be $l_{+}=\gamma_{+} \cos \theta_{2}^{(+,+)}$and $l_{-}$ $=\gamma_{-} \cos \theta_{2}^{(+,-)}$. As $\chi>0, \beta_{+}>\beta_{-}$and $\gamma_{+}>\gamma_{-}$so, from Eq. (31), $\theta_{1}^{(+,-)}>\theta_{1}^{(+,+)}$and $\theta_{2}^{(+,-)}>\theta_{2}^{(+,+)}$. A similar discussion can be developed when the incident wave is the core's LCP characteristic wave, as depicted in Fig. 2(b), and where $\theta_{1}^{(-)}=\theta_{1}^{(-,-)}$, corresponding to

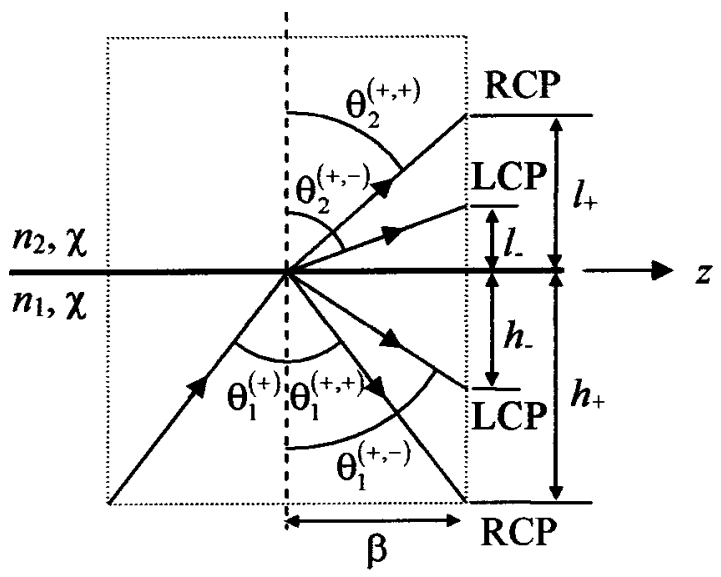

(a)

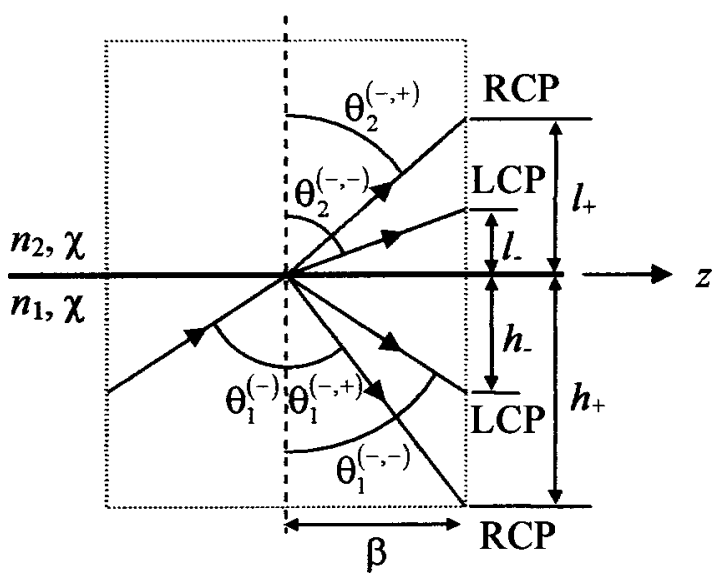

(b)

Fig. 2. Trajectories of rays at a planar interface between different chiral media when the incident wave is (a) a RCP wave and (b) a LCP wave.

$$
\begin{aligned}
\beta=\beta_{-} \sin \theta_{1}^{(-)} & =\beta_{+} \sin \theta_{1}^{\left(-,{ }^{+}\right)} \\
& =\gamma_{+} \sin \theta_{2}^{\left(-,{ }^{+}\right)}=\gamma_{-} \sin \theta_{2}{ }^{(-,-)} .
\end{aligned}
$$

We should stress again that we can write Eqs. (31) and (32) as two independent equations as a result of our simplifying hypothesis of a planar core-cladding interface that allows us to treat these two cases separately.

When the incident wave from the core is the RCP wave, two critical angles will exist: $\theta_{1}{ }^{(+)}=\theta_{1 c}{ }^{\left(+,{ }^{+}\right)}$, where $\sin \theta_{1 c}{ }^{(+,+)}=\gamma_{+} / \beta_{+}$[corresponding to $\theta_{2}{ }^{\left(+,{ }^{+}\right)}=\pi / 2$ ], and $\theta_{1}{ }^{(+)}=\theta_{1 c}{ }^{(+,-)}$, where $\sin \theta_{1 c}{ }^{(+,-)}=\gamma_{-} / \beta_{+}$[corresponding to $\left.\theta_{2}{ }^{(+,-)}=\pi / 2\right]$. Likewise, when a LCP wave is incident, we will have two critical angles: $\theta_{1}{ }^{(-)}$ $\left.=\theta_{1 c}{ }^{(-,}{ }^{+}\right)$, where $\sin \theta_{1 c^{(-,}}{ }^{+)}=\gamma_{+} / \beta_{-}$[corresponding to $\left.\left.\theta_{2}{ }^{(-,}+{ }^{+}\right)=\pi / 2\right]$, and $\left.\theta_{1}^{(-)}=\theta_{1 c}{ }^{(-,},-\right)$where $\sin \theta_{1 c}{ }^{(-,-)}$ $=\gamma_{-} / \beta_{-}$[corresponding to $\left.\theta_{2}{ }^{\left(-,{ }^{-}\right)}=\pi / 2\right]$. In Table 1 we summarize the four critical angles that are necessary to our physical discussion. Note that, according to Eq (29), one has $\left(n_{1}-\chi\right)\left(n_{2}+\chi\right)>\left(n_{1}+\chi\right)\left(n_{2}-\chi\right)$, leading to $\beta_{-} \gamma_{+}>\beta_{+} \gamma_{-}$, and hence

$$
\theta_{1 c}{ }^{(-,+)}>\theta_{1 c}{ }^{(+,+)}>\theta_{\left.1 c^{(-,},-\right)}>\theta_{1 c^{(+,-)}} .
$$

Therefore, for an incident RCP wave, both of the core's characteristic waves undergo total internal reflection as long as $\theta_{1}{ }^{(+)} \geqslant \theta_{1 c}{ }^{(+,+)}>\theta_{1 c}{ }^{(+,-)}$. In this case a sur- 
face mode will result, with $\theta_{2}^{(+, \pm)}=\pi / 2-i \vartheta_{+}$(with $\left.\vartheta_{+}>0\right)$. In fact, according to Eq. (31), $\beta=\gamma_{+} \cosh \vartheta_{+}$ and, in the cladding, $l_{ \pm}=i \alpha_{ \pm}$, where $\alpha_{ \pm}=\gamma_{ \pm} \sinh \vartheta_{ \pm}$. The core's RCP wave is transmitted (i.e., $l_{+}>0$ ), whereas the core's LCP wave undergoes total internal reflection (i.e., $l_{-}=i \alpha_{-}$) if, for an incident RCP wave, $\theta_{1 c}{ }^{\left(+,{ }^{+}\right)}>\theta_{1}{ }^{(+)} \geqslant \theta_{1 c}{ }^{(+,-)}$. Likewise, for an incident LCP wave, the core's RCP wave is transmitted and the core's LCP wave undergoes total internal reflection if $\left.\theta_{1 c}{ }^{\left(-,{ }^{+}\right)}>\theta_{1}{ }^{(-)} \geqslant \theta_{1 c}{ }^{(-,},-\right)$. Because the core's characteristic waves are actually coupled, a semileaky mode will result if $\theta_{1 c}{ }^{\left(+,{ }^{+}\right)}>\theta_{1} \geqslant \theta_{1 c}{ }^{(-,-)}$. So the cutoff of a surface wave will always occur for $\beta=\beta_{+} \sin \theta_{1 c}{ }^{(+,+)}=\gamma_{+}$, and hence a semileaky wave is always present as long as $q_{-} \leqslant n_{\text {eff }}<q_{+}$. If $\theta_{1}<\theta_{1 c^{(-)}}{ }^{-)}$, both of the core's characteristic waves are transmitted into the cladding, and hence $l_{+}>0$ as well as $l_{-}>0$. Therefore, if $n_{\text {eff }}<q_{-}$, only completely unguided modes can exist.

In Fig. 3 we represent, in the plane $\left(\chi, n_{\text {eff }}\right)$, the two regions where guided waves can occur: region 1 for surface waves and region 2 for semileaky waves. One should note that $\theta_{1}^{(+)}=\pi / 2$ is the maximum angle in Eq. (31); hence one should always have $n_{\text {eff }}<p_{+}$. A surface wave can become a semileaky wave if $\chi>\chi_{c}$, where the critical value $\chi_{c}$ is such that $n_{\text {eff }}=q_{+}$and hence $\chi_{c}=n_{\text {eff }}$ $-n_{2}$. Therefore, for a semileaky wave, $\alpha_{ \pm}$given in Eq. (12a) will be complex too. One can easily see that, in this case,

$$
\begin{aligned}
\mathfrak{R}\left(\alpha_{+}\right) \mathfrak{I}\left(\alpha_{+}\right) & =\mathfrak{R}\left(\alpha_{-}\right) \mathfrak{I}\left(\alpha_{-}\right), \\
\mathfrak{R}^{2}\left(\alpha_{+}\right)-\mathfrak{I}^{2}\left(\alpha_{+}\right) & =\mathfrak{R}^{2}\left(\alpha_{-}\right)-\mathfrak{I}^{2}\left(\alpha_{-}\right)-4 n_{2} \chi k_{0}{ }^{2} .
\end{aligned}
$$

Table 1. Critical Angles at the Core-Cladding Interface (Fig. 2)

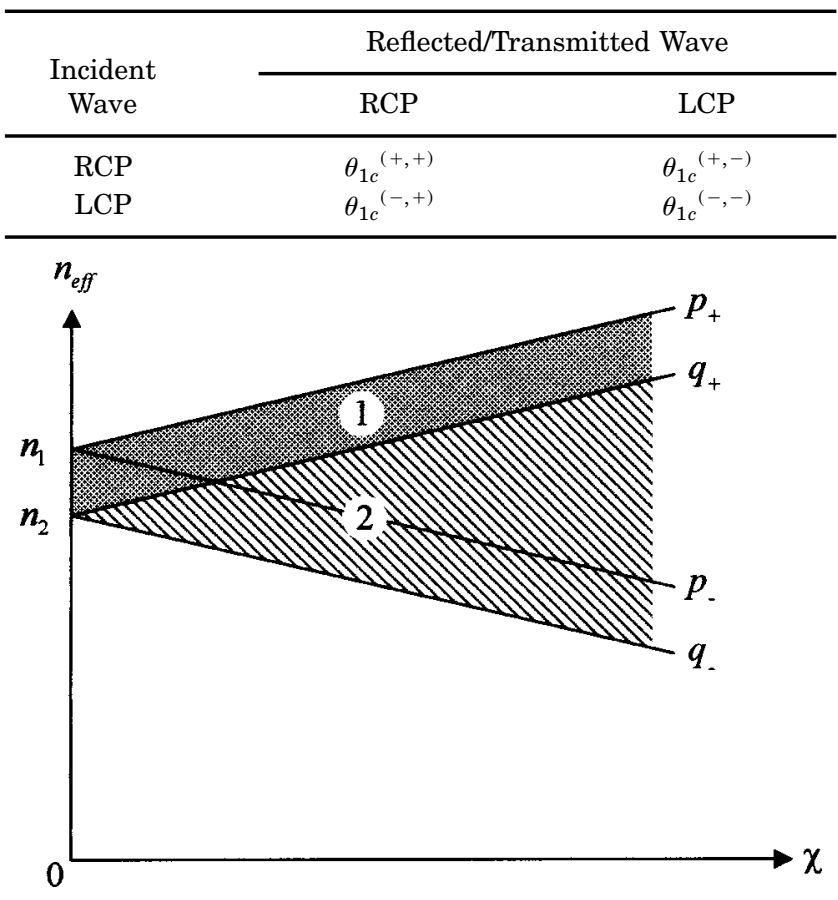

Fig. 3. Effective refractive index $n_{\text {eff }}$ versus chirality $\chi$. In region 1 , only surface modes may propagate, whereas in region 2 only semileaky modes may exist.
In fact, as $\alpha_{+}{ }^{2}-\alpha_{-}{ }^{2}=\gamma_{-}{ }^{2}-\gamma_{+}{ }^{2}=-4 n_{2} \chi k_{0}{ }^{2}<0$, one should have $\mathfrak{I}\left(\alpha_{+}{ }^{2}-\alpha_{-}{ }^{2}\right)=0$, thus leading to Eqs. (34). According to Eq. (18), if $r \rightarrow \infty$, one has the following asymptotic representation ${ }^{51}$ :

$$
F(r) \sim \sqrt{\frac{\pi}{2}}\left[\frac{B_{+}}{\sqrt{\alpha_{+} r}} \exp \left(-\alpha_{+} r\right)+\frac{B_{-}}{\sqrt{\alpha_{-} r}} \exp \left(-\alpha_{-} r\right)\right] .
$$

For a semileaky mode the RCP component is the transmitted characteristic wave that gives rise to leakage and hence, in $\exp \left(-\alpha_{+} r\right)=\exp \left[-\mathfrak{R}\left(\alpha_{+}\right) r\right] \exp \left[-i \Im\left(\alpha_{+}\right) r\right]$, one should have $\mathfrak{R}\left(\alpha_{+}\right)<0$ and $\Im\left(\alpha_{+}\right)<0$, corresponding to an outgoing wave. However, for the LCP component, one should have $\mathfrak{R}\left(\alpha_{-}\right)>0$ and hence, according to Eq. (34a), $\Im\left(\alpha_{-}\right)>0$, corresponding to an incoming wave. One should always bear in mind that, in a semileaky mode, the RCP and LCP components are coupled and do not represent waves propagating separately: Only the superposition of RCP and LCP components plays an independent role in the whole picture.

In summary: A semileaky wave always occurs whenever only the core's LCP wave undergoes total internal reflection; in other words, it is the core's RCP wave that is always responsible for leakage, as that is the only characteristic wave of the core that is transmitted. For a surface wave we always have, according to Eq. (31), $q_{+}$ $\leqslant n_{\text {eff }}<p_{+}$, whereas, for a semileaky wave, $q_{-} \leqslant n_{\text {eff }}$ $<q_{+}$. One should finally note that, if $\chi<0$, the roles of the core's two characteristic waves would be interchanged.

\section{NUMERICAL RESULTS}

In this section we present several numerical results that show that both surface and semileaky modes can propagate in chiral optical fibers. All these numerical results are solutions of Eq. (28)—our modal equation. We stress, however, that any algorithm used to find the numerical solutions of Eq. (28) should be able to include, according to Eq. (30), complex values. Furthermore, as we established in Section 3, semileaky modes correspond to special complex values such that

$$
\begin{array}{ll}
\mathfrak{R}\left(w_{+}\right)<0, & \Im\left(w_{+}\right)<0, \\
\mathfrak{R}\left(w_{-}\right)>0, & \Im\left(w_{-}\right)>0 .
\end{array}
$$

In fact, we have $\alpha=0$ and hence $n_{\text {eff }}=\beta / k_{0}$ for surface modes only. Therefore the numerical algorithm has to carefully choose the appropriate Riemann sheets associated with

$$
w_{ \pm}=\frac{2 \pi a}{\lambda}\left[\left(n_{\mathrm{eff}}+i \frac{\alpha \lambda}{4 \pi}\right)^{2}-q_{ \pm}^{2}\right]^{1 / 2}
$$

by taking branch points and branch cuts into account. We have used a method that finds all the roots of an analytical function within a circle of a prescribed radius. ${ }^{52}$

The parameters used in our numerical results are typical of an optical fiber operated in the third window. Therefore for all numerical results the values $n_{1}=1.48$ and $n_{2}=1.46$, with $\mu_{1}=\mu_{2}=1$ and $\chi_{1}=\chi_{2}=\chi$, were adopted. Moreover, the core radius is $a=4.58 \mu \mathrm{m}$ 


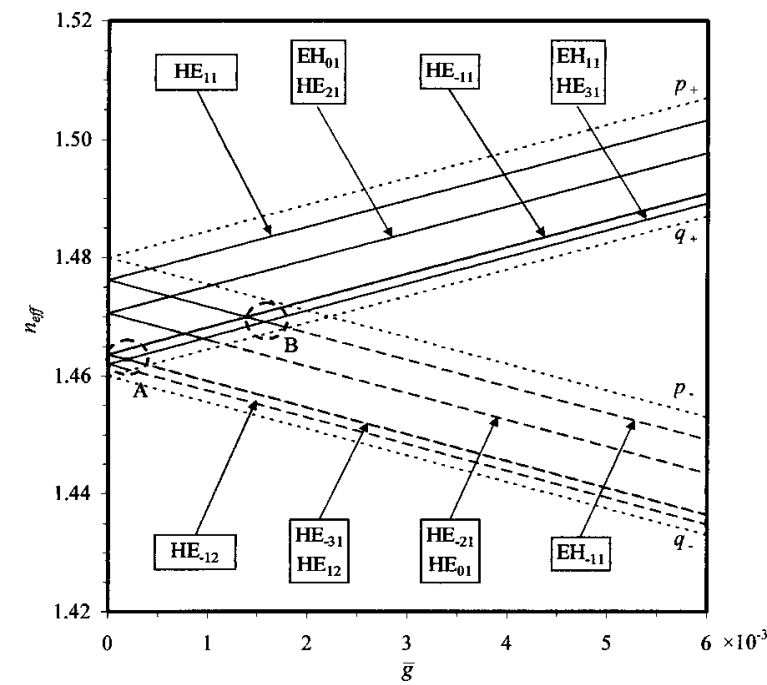

Fig. 4. Effective refractive index versus the fiber's normalized chirality parameter for all modes propagating at $v=4.5$. Arrows represent the normalized propagation constants for unbounded media. Solid lines, surface modes; dashed lines, semileaky modes. Modes $\mathrm{HE}_{12}$ and $\mathrm{EH}_{11}$ couple in region $\mathrm{A}$, whereas modes $\mathrm{EH}_{-11}$ and $\mathrm{HE}_{-11}$ couple in region $\mathrm{B}$ (see Figs. 5 and 6 below).

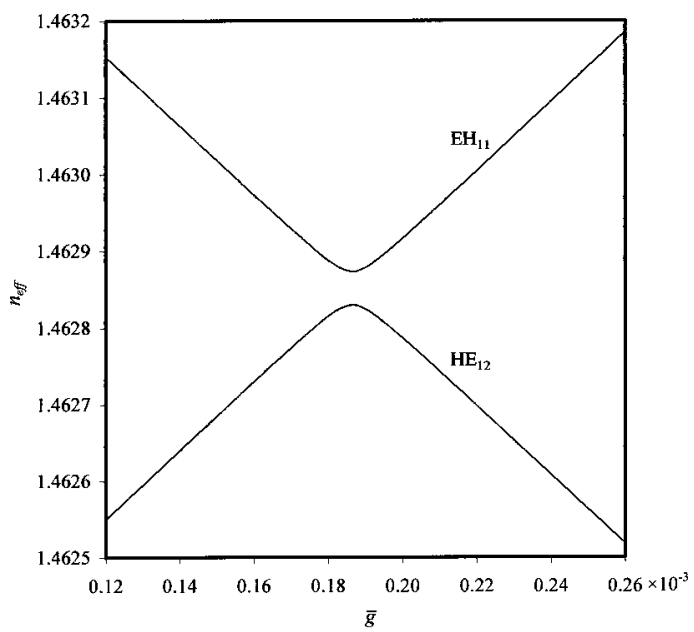

Fig. 5. Coupling between $\mathrm{EH}_{11}$ and $\mathrm{HE}_{12}$ modes.

throughout, leading to a normalized value $v=4.5$, with $v=2 \pi a \sqrt{{n_{1}}^{2}-n_{2}{ }^{2}} / \lambda$, at wavelength $\lambda=1.55 \mu \mathrm{m}$. One should also note that, according to Eq. (5), one has

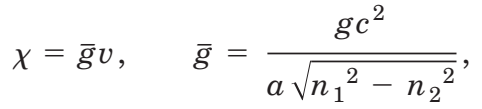

where the new normalized value $\bar{g}$ was introduced. The values adopted for parameter $\bar{g}$ are within a range that is bounded according to the condition $|\chi|<\sqrt{\epsilon \mu}$ for lossless chiral media. ${ }^{47}$

As was already pointed out in the analytical formulation, there are no $\mathrm{TE}_{0 n}$ or $\mathrm{TM}_{0 n}$ modes, unlike for achiral optical fibers in which these transverse modes with no azimuthal variation can propagate. In fact, owing to the magnetoelectric nature of chiral media, only $\mathrm{HE}_{0 n}$ or $\mathrm{EH}_{0 n}$ hybrid modes can propagate when there is no field variation with coordinate $\phi$ (i.e., when $m=0$ ). Further- more, the modal equation is no longer an even function of azimuthal wave number $m$ : For chiral fibers, $m=+j$ and $m=-j$ correspond to two different sets of hybrid modes, labeled $\mathrm{HE}_{j n}$ (or $\mathrm{EH}_{j n}$ ) and $\mathrm{HE}_{-j n}\left(\right.$ or $\mathrm{EH}_{-j n}$ ), respectively. Only for the achiral case do these two sets of hybrid modes become degenerate, as the modal equation is, for $\bar{g}=0$, an even function of azimuthal wave number $m$.

One should stress that the mode designations adopted herein are the following: Any given hybrid mode is labeled $\mathrm{HE}_{m n}$ or $\mathrm{EH}_{m n}$ according to its characteristics in the achiral limit (i.e., when $\bar{g} \rightarrow 0$ ). In Fig. 4 we show the effective refractive index $n_{\text {eff }}=\mathfrak{R}(\beta) / k_{0}$ as a function of the fiber's normalized chirality parameter $\bar{g}$ for all propagating modes. The dotted lines represent the normalized propagation constants for unbounded media as defined in Eqs. (12b) and (12c). Solid lines represent completely guided (or surface) modes, and dashed lines represent semileaky modes. All hybrid modes are, obviously, surface modes in the achiral limit. Modes for which $n_{\text {eff }}$ is an increasing function of $\bar{g}$ are always surface modes. Modes for which $n_{\text {eff }}$ is a decreasing function of $\bar{g}$, however, turn into semileaky modes when $n_{\text {eff }}$ crosses the $q_{+}$line (i.e., when its RCP component starts radiating energy). Therefore, in Fig. 4 all modes labeled at the top are surface modes, whereas those labeled at the bottom become semileaky for a critical value $\bar{g}_{c}$ of the fiber's normalized chirality parameter. Every semileaky mode has its specific $\bar{g}_{c}$ value.

A new effect that is not available in common isotropic fibers is also shown in Fig. 4: Hybrid modes with the same azimuthal wave number $m$ may couple. Indeed, in Fig. 4 there is a coupling between $\mathrm{HE}_{12}$ and $\mathrm{EH}_{11}$ modes in region $\mathrm{A}$ and a coupling between $\mathrm{EH}_{-11}$ and $\mathrm{HE}_{-11}$ modes in region B. In Figs. 5 and 6 these two coupling regions of Fig. 4 are shown in more detail. One should note, however, that, because of these couplings, the mode designation for chiral fibers does not keep its usual meaning: Whenever coupling between two hybrid modes occurs, the original significance of mode labeling is overridden.

For semileaky modes a complex longitudinal wave number $\beta$ has to be considered, as stated in inequalities

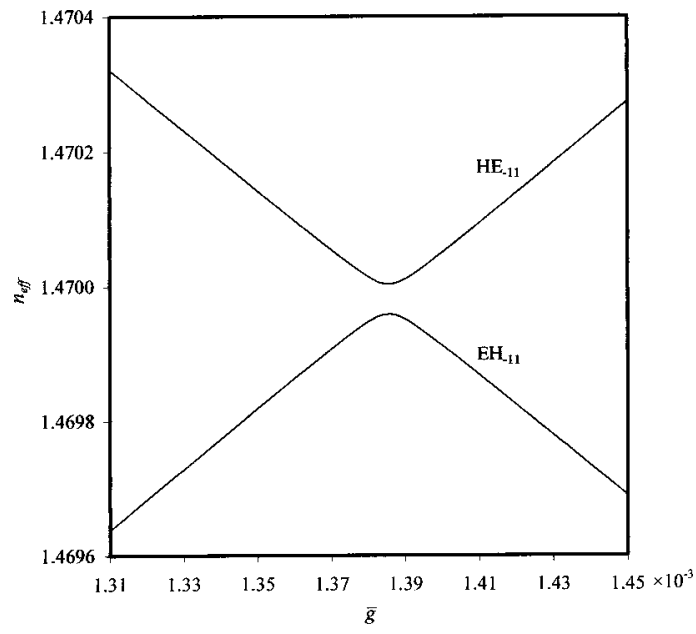

Fig. 6. Coupling between $\mathrm{EH}_{-11}$ and $\mathrm{HE}_{-11}$ modes. 


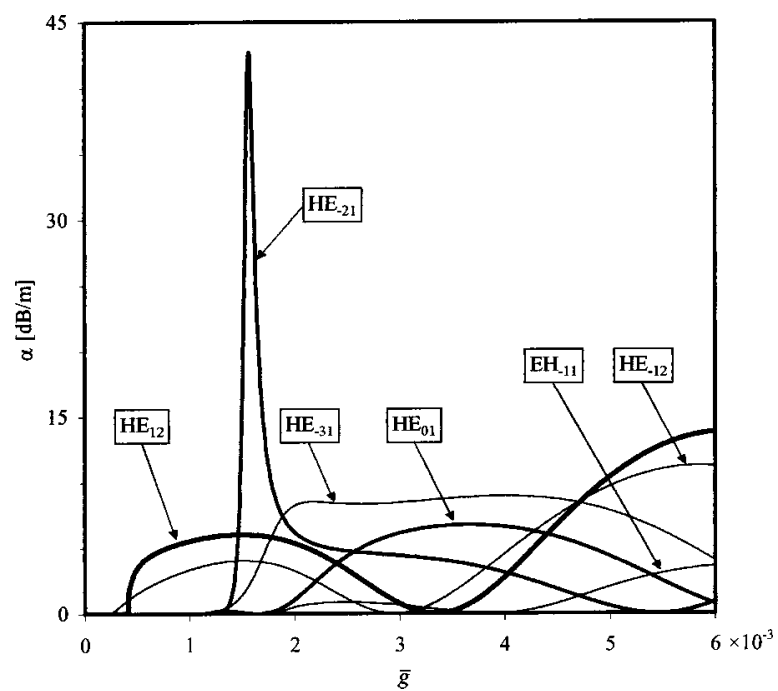

Fig. 7. Leakage loss versus chirality for the semileaky modes in Fig. 4. Surface modes become semileaky modes when the fiber's normalized chirality parameter is above a certain critical value that depends on each individual mode.

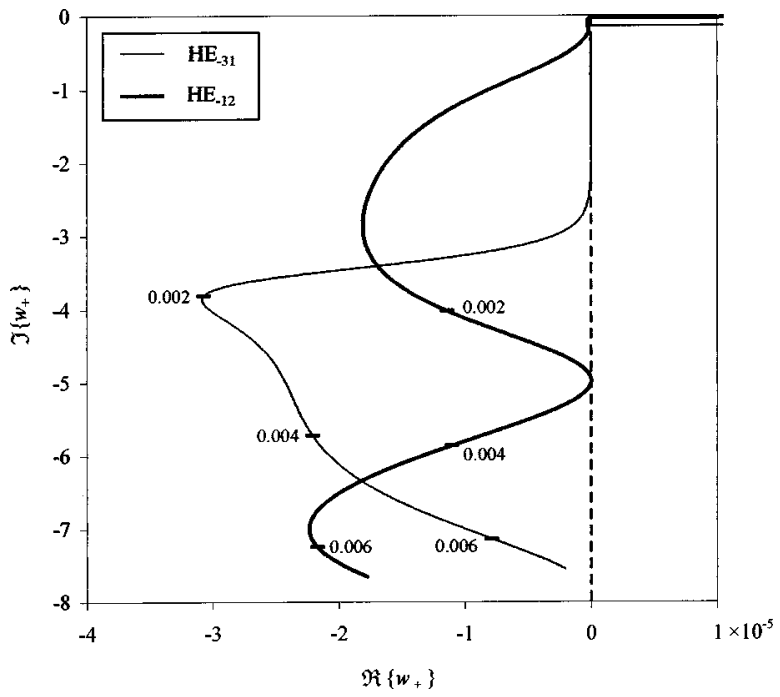

Fig. 8. Root loci of normalized variable $w_{+}$for $\mathrm{HE}_{-31}$ and $\mathrm{HE}_{-12}$ modes in terms of normalized parameter $\bar{g}$.

(36) and Eq. (37). In Fig. 7 we show $\alpha$ (in decibels per meter) as a function of the fiber's normalized chirality parameter $\bar{g}$ for all semileaky modes in Fig. 4 . The values found for this leakage loss can be severely high, so this new effect should not be disregarded in chiral optical fibers. Finally, in Fig. 8 we present, as an illustrative example, a root-locus plot for the complex solutions $w_{+}$of two semi-leaky modes to bring out the conditions expressed through inequalities (36).

\section{CONCLUSIONS}

A general theory of guided waves in optical fibers in which both core and cladding are chiral media has been derived. It was shown that, in chiral optical fibers, both surface and semileaky modes can propagate. This new leakage effect in chiral optical fibers, which is associated with semileaky modes, was physically discussed, and several numerical results were presented for both surface and semileaky waves. Although only the case in which both core and cladding are chiral media was physically and numerically considered in this paper, one should note that semileaky modes also occur when only the cladding is chiral (i.e., the core may be a common isotropic medium with $\chi_{1}=0$ as long as $\chi_{2} \neq 0$ ). The continuous spectrum of radiation modes for chiral optical fibers was not analyzed in this paper.

The key feature that makes leakage possible is the existence of two coupled characteristic waves in the cladding: Whenever only one of the core's characteristic waves undergoes total internal reflection, a semileaky mode is generated. Hence other kinds of optical fiber with birefringent media, such as anisotropic optical fibers, should also exhibit this behavior.

A complex longitudinal wave number has to be considered for every semileaky mode: The real part accounts for guidance (to account for the partial internal reflection at the core-cladding interface) and the imaginary part accounts for leakage (to account for the energy radiated through the core's characteristic wave that is transmitted). Therefore complex solutions from properly specified Riemann sheets should be sought when one is solving the modal equation for an optical fiber with a chiral cladding. That is the reason why this leakage effect has been systematically disregarded in the literature so far: Previous studies of chiral optical fibers were looking exclusively for surface waves, and hence only real roots were found when the corresponding modal equation was solved.

Common isotropic (or achiral) optical fibers are now well-known devices in optical science and technology. Nevertheless, new materials and effects are under current investigation. From the designer's point of view, chiral optical fibers may increase flexibility in optimization procedures because of the extra chirality parameter. Still, further developments in artificially produced chiral materials-namely, for applications at optical frequencies-are needed.

F. M. Janeiro's e-mail address is fernando.janeiro @lx.it.pt.

\section{REFERENCES}

1. A. Lakhtakia, ed., Selected Papers on Natural Optical Activity, Vol. MS15 of SPIE Milestone Series (SPIE Optical Engineering Press, Bellingham, Wash. 1990).

2. I. V. Lindell, A. H. Sihvola, and J. Kurkijärvi, "Karl F. Lindman - the last Hertzian and a harbinger of electromagnetic chirality," IEEE Antennas Propag. Mag. 34, 24-30 (1992)

3. D. L. Jaggard, A. R. Mickelson, and C. H. Papas, "On electromagnetic waves in chiral media," J. Appl. Phys. 18, 211216 (1979).

4. S. A. Kuehl, S. S. Grové, E. Kuehl, M. Bingle, and J. H. Cloete, "Manufacture of microwave chiral materials and their electromagnetic properties," in Advances in Complex Electromagnetic Materials, A. Priou, A. Sihvola, S. Tretyakov, and A. Vinogradov, eds. (Kluwer Academic, Dordrecht, The Netherlands, 1997), pp. 317-332.

5. H. Cory, "Chiral devices-an overview of canonical problems," J. Electromagn. Waves Appl. 9, 805-829 (1995).

6. I. V. Lindell, A. H. Sihvola, S. A. Tretyakov, and A. J. Viitanen, Electromagnetic Waves in Chiral and Bi-Isotropic Media (Artech House, Boston, Mass., 1994), pp. 119-151. 
7. C. R. Paiva and A. M. Barbosa, "A method for the analysis of biisotropic planar waveguides-application to a grounded chiroslabguide," Electromagnetics 11, 209-221 (1991).

8. H. Cory and I. Rosenhouse, "Electromagnetic wave propagation along a chiral slab," IEE Proc. H 138, 51-54 (1991)

9. M. I. Oksanen, P. K. Koivisto, and I. V. Lindell, "Dispersion curves and fields for a chiral slab waveguide," IEE Proc. H 138, 327-334 (1991).

10. N. Engheta and P. Pelet, "Surface waves in chiral layers," Opt. Lett. 16, 723-725 (1991).

11. C. R. Paiva, A. L. Topa, and A. M. Barbosa, "Semileaky waves in dielectric chirowaveguides," Opt. Lett. 17, 16701672 (1992)

12. A. L. Topa, C. R. Paiva, and A. M. Barbosa, "Least squares boundary residual method for the analysis of step discontinuities in open chiral waveguides," Int. J. Electron. Commun. 55, 281-291 (2001).

13. J. A. M. Svedin, "Propagation analysis of chirowaveguides using the finite-element method," IEEE Trans. Microwave Theory Tech. 38, 1488-1496 (1990).

14. H. Cory and T. Tamir, "Coupling processes in circular open chirowaveguides," IEE Proc. H 139, 165-170 (1992).

15. H. Cory and S. Gov, "Mode energy transfer along a circular open chirowaveguide," Microwave Opt. Technol. Lett. 6, 536-541 (1993).

16. A. K. Singh, Kh. S. Singh, P. Khastgir, S. P. Ojha, and O. N. Singh, "Modal cutoff condition of an optical chiral fiber with different chiralities in the core and the cladding," J. Opt. Soc. Am. B 11, 1283-1287 (1994).

17. R. C. Qiu and I-T. Lu, "Guided waves in chiral optical fibers,” J. Opt. Soc. Am. A 11, 3212-3219 (1994).

18. S. F. Mahmoud, "Guided modes on open chirowaveguides," IEEE Trans. Microwave Theory Tech. 43, 205-209 (1995).

19. F. M. Janeiro, A. L. Topa, and C. R. Paiva, "Semi-leaky modes in chiral optical fibers," presented at LEOS 2001, 14th Annual Meeting, San Diego, Calif., 2001.

20. S. T. Peng and A. A. Oliner, "Guidance and leakage properties of a class of open dielectric waveguides. I. Mathematical formulations," IEEE Trans. Microwave Theory Tech. MTT-29, 843-855 (1981).

21. A. A. Oliner, S. T. Peng, T. I. Hsu, and A. Sanchez, "Guidance and leakage properties of a class of open dielectric waveguides. II. New physical effects," IEEE Trans. Microwave Theory Tech. MTT-29, 855-869 (1981).

22. K. Yamanouchi, T. Kamiya, and K. Shibayama, "New leaky surface waves in anisotropic metal-diffused optical waveguides," IEEE Trans. Microwave Theory Tech. MTT26, 298-305 (1978).

23. S. K. Sheem, W. K. Burns, and A. F. Milton, "Leaky mode propagation in Ti-diffused $\mathrm{LiNbO}_{3}$ and $\mathrm{LiTaO}_{3}$ waveguides," Opt. Lett. 3, 76-78 (1978).

24. J. Čtyroký and M. Ćada, "Guidance and semileaky modes in anisotropic optical waveguides of $\mathrm{LiNbO}_{3}$ type," Opt. Commun. 27, 353-357 (1978)

25. D. Marcuse and I. P. Kaminov, "Modes of a symmetric slab optical waveguide in birefringent media. II. Slab with a coplanar optical axis," IEEE J. Quantum Electron. QE-15, 92-101 (1979).

26. W. K. Burns, S. K. Sheem, and A. F. Milton, "Approximate calculation of leaky-mode loss coefficients for Ti-diffused $\mathrm{LiNbO}_{3}$ waveguides," IEEE J. Quantum Electron. QE-15, 1282-1289 (1979)

27. J. Čtyroký and M. Čada, "Generalized WKB method for the analysis of light propagation in inhomogeneous anisotropic optical waveguides," IEEE J. Quantum Electron. QE-17, 1064-1070 (1981).

28. M. Koshiba, H. Kumagami, and M. Suzuki, "Finite-element solution of planar arbitrary anisotropic diffused optical waveguides," J. Lightwave Technol. LT-3, 773-778 (1985).

29. A. Knoesen, T. K. Gaylord, and M. G. Moharam, "Hybrid guided modes in uniaxial dielectric planar waveguides," J. Lightwave Technol. 6, 1083-1104 (1988).

30. L. Torner, F. Canal, and J. H. Marco, "Leaky modes in multilayer uniaxial optical waveguides," Appl. Opt. 29, 2805-2814 (1990).

31. L. Torner, J. Recolons, and J. P. Torres, "Guided-to-leaky mode transition in uniaxial optical slab waveguides," J. Lightwave Technol. 11, 1592-1600 (1993).

32. R. E. Smith and S. N. Houde-Walter, "The migration of bound and leaky solutions to the waveguide dispersion relation,” J. Lightwave Technol. 11, 1760-1768 (1993).

33. A. D'Orazio, M. De Sario, V. Petruzzelli, and F. Prudenzano, "Leaky wave propagation in planar multilayer birefringent waveguides: longitudinal dielectric tensor configurations," J. Lightwave Technol. 12, 453-462 (1994).

34. T. A. Maldonado and T. K. Gaylord, "Hybrid guided modes in biaxial planar waveguides," J. Lightwave Technol. 14, 486-499 (1996).

35. R. E. Collin, Field Theory of Guided Waves, 2nd ed., (IEEE Press, New York, 1991), pp. 725-744.

36. E. U. Condon, "Theory of optical rotatory power," Rev. Mod. Phys. 9, 432-457 (1937).

37. A. H. Sihvola and I. V. Lindell, "Bi-isotropic constitutive relations," Microwave Opt. Technol. Lett. 4, 295-297 (1991).

38. A. Lakhtakia, Beltrami Fields in Chiral Media (World Scientific, Singapore, 1994).

39. A. Lakhtakia and W. S. Weiglhofer, "Are linear, nonreciprocal, biisotropic media forbidden?" IEEE Trans. Microwave Theory Tech. 42, 1715-1716 (1994).

40. A. Lakhtakia and W. S. Weiglhofer, "Constraint on linear, homogeneous constitutive relations," Phys. Rev. E 50, 5017-5019 (1994)

41. W. S. Weiglhofer and A. Lakhtakia, "A brief review of a new development for constitutive relations for linear bianisotropic media," IEEE Antennas Propag. Mag. 37, 32-35 (1995).

42. W. S. Weiglhofer and A. Lakhtakia, "The Post constraint revisited," Int. J. Electron. Commun. 52, 276-279 (1998).

43. E. J. Post, Formal Structure of Electromagnetics-General Covariance and Electromagnetics (Dover, Mineola, N.Y., 1997), pp. 129, 168-171.

44. A. H. Sihvola, "Are nonreciprocal bi-isotropic media forbidden indeed?" IEEE Trans. Microwave Theory Tech. 43, 2160-2162 (1995)

45. S. Tretyakov, "Anything wrong with the naturally nonreciprocal materials?" IEEE Antennas Propag. Mag. 38, 84-85 (1996)

46. J. C. Monzon, "Author's reply," IEEE Trans. Antennas Propag. 45, 749 (1997)

47. I. V. Lindell, A. H. Sihvola, S. A. Tretyakov, and A. J. Viitanen, Electromagnetic Waves in Chiral and Bi-Isotropic Media (Artech House, Boston, Mass., 1994), pp. 23-58.

48. A. Lakhtakia, "Recent contributions to classical electromagnetic theory of chiral media: what next?" Speculations Sci. Technol. 14, 2-17 (1991).

49. M. J. Adams, An Introduction to Optical Waveguides (Wiley, Chichester, UK, 1981), pp. 223-228.

50. A. W. Snyder and J. D. Love, Optical Waveguide Theory (Chapman \& Hall, London, 1983), pp. 248-259.

51. M. Abramowitz and I. A. Stegun, eds., Handbook of Mathematical Functions (Dover, New York, 1965), Eq. (9.7.2).

52. M. P. Carpentier and A. F. dos Santos, "Solution of equations involving analytic functions," J. Comput. Phys. 45, $210-220$ (1982). 